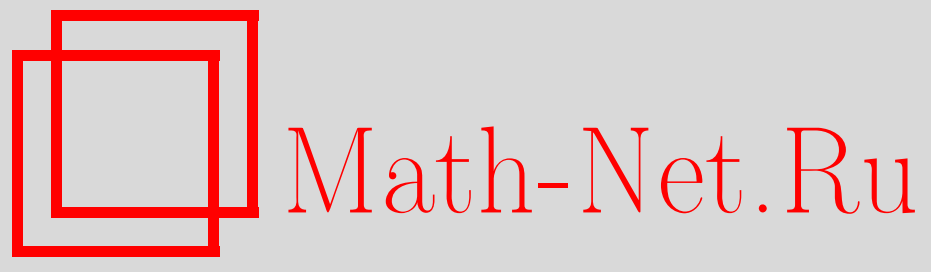

А. Л. Лукашов, Дополнения к принципу гармонической меры Р. Неванлинны, Матем. заметки, 2008, том 84, выпуск 4, 632-633

DOI: https://doi.org/10.4213/mzm4514

Использование Общероссийского математического портала Math-Net.Ru подразумевает, что вы прочитали и согласны с пользовательским соглашением http://www . mathnet.ru/rus/agreement

Параметры загрузки:

IP: 3.95 .254 .165

26 апреля 2023 г., 18:05:47

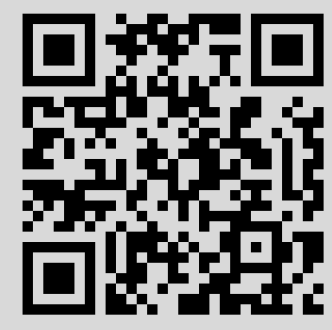




\section{Дополнения к принципу гармонической меры Р. Неванлинны}

\section{А. Л. Лукашов}

Хорошо известен (см., например, [1], [2]) принцип гармонической меры, который при ряде условий утверждает, что гармоническая мера образа части границы области не меньше гармонической меры прообраза.

Цель данной заметки - привести два утверждения, которые дают "обратные" оценки. Всюду в дальнейшем $\omega$ обозначает гармоническую меру соответствующей части границы области относительно данной точки области.

Теорема 1. Пусть $D$ и $G$-области в $\overline{\mathbb{C}}$, ограниченные конечным числом жордановых дуг. Если $w(z)$ - мероморфная в $D$, непрерывная в $\bar{D}$ функиия, производная которой не обращается в нуль в $D, w(D) \supset G u w(\partial D)=\partial G$, то для любого множеств $\alpha_{z} \subset \partial D$, состоящего из конечного числа дуг, и для любого $w_{0} \in G$ выполнено

$$
\omega\left(w_{0}, \alpha_{w}, G\right) \leqslant \sum_{k} \omega\left(z_{k}\left(w_{0}\right), \alpha_{z}, D\right),
$$

где суммирование ведется по всевозможным решениям $z_{k}$ уравнения $w(z)=w_{0} u \alpha_{w}=$ $w\left(\alpha_{z}\right)$.

ДоказатеЛьство. Пусть $w_{0} \in G$. Тогда уравнение

$$
f(z)=w_{0}
$$

имеет лишь конечное число решений в $D$. В самом деле, в противном случае для предельной точки $z_{0}$ решений уравнения (1) имеем $z_{0} \in \partial D$ и $f\left(z_{0}\right)=w_{0}$, что противоречит условию $f(\partial D)=\partial G$. Докажем, что число решений уравнения (1) одно и то же для всех $w_{0} \in G$. Соединим две произвольные точки $w_{1}$ и $w_{2}$ области $G$ кривой, лежащей в $G$. Для каждой точки $w$ этой кривой можно найти такую ее окрестность $V$, которая является образом дизъюнктного объединения окрестностей $U_{j}$ решений уравнения $f(z)=w$ при отображении $f$, причем $f$ взаимно однозначно на каждом из $U_{j}$ (в силу того, что $f^{\prime}(z)$ не обращается в нуль). В силу компактности кривой можно выбрать конечное число таких окрестностей $V$, покрывающих эту кривую. Так как при переходе от одной окрестности к другой листность отображения не изменяется, то число решений уравнений $f(z)=w_{1}$ и $f(z)=w_{2}$ одно и то же. Поэтому функция

$$
h(w)=\sum_{k} \omega\left(z_{k}(w), \alpha_{z}, D\right)-\omega\left(w, \alpha_{w}, G\right)
$$

является однозначной гармонической в $G$. Оценим ее предельные значения на $\partial G$. Пусть $\widetilde{w} \in \alpha_{w}$. Тогда найдется по крайней мере одно значение $z \in \alpha_{z}$ такое, что $w(z)=\widetilde{w}$. Следовательно,

$$
\lim _{\substack{w \rightarrow \widetilde{w} \\ w \in G}} h(w) \geqslant 0 .
$$

Если $\widetilde{w} \in \partial G \backslash \alpha_{w}$, то $\omega\left(\widetilde{w}, \alpha_{w}, G\right)=0$ и неравенство (2) также имеет место. Из (2) по принципу минимума для гармонических функций следует требуемое.

Теорема 2. Пусть области $D$ и $G$ ограничены конечным числом замкнутых жордановых кривых, область $D$ имеет на своей граниче открытую аналитическую дугу $\gamma$, область $G$ имеет на своей гранище такую же дугу $\Gamma$, причем $f(\gamma)=\Gamma$ и $f(\partial D) \subset \partial G$

Работа выполнена при поддержке Российского фонда фундаментальных исследований (грант № 07-01-00167).

(C) А. Л. ЛУкашов, 2008 
для рачиональной функции $f(z)$. Тогда для любой точки $w_{0} \in f(D) \cap G$ справедливо неравенство

$$
\omega\left(w_{0}, \Gamma, G\right) \leqslant \sum_{k} \omega\left(z_{k}\left(w_{0}\right), \gamma, D\right),
$$

причем суммирование ведется по всевозможным решениям $z_{k}$ уравнения $f(z)=w_{0}$ (с учетом их кратностей).

ДоказАтельство. Пусть сначала $w_{0}=\infty$. Тогда функция $f(z)$ удовлетворяет условиям следствия 2 из [3] и для всех $z \in \gamma$ справедливо неравенство

$$
\left|f^{\prime}(z)\right| \frac{\partial g_{G}(f(z), \infty)}{\partial n} \leqslant \sum_{k} \frac{\partial g_{D}\left(z, z_{k}(\infty)\right)}{\partial n},
$$

где $\partial / \partial n$ означает дифференцирование вдоль внутренней нормали к соответствующей граничной дуге, $g$ - функция Грина соответствующей области.

Учитывая представление гармонической меры [1], имеем

$$
\omega(\infty, \Gamma, G)=\frac{1}{2 \pi} \int_{\Gamma} \frac{\partial g_{G}(\zeta, \infty)}{\partial n}|d \zeta|
$$

Так как $f(\gamma)=\Gamma$, то $\gamma$ можно разбить на конечное число дуг $\gamma_{j}$ таких, что на каждой из дуг $\gamma_{j}$ функция $f(z)$ осуществляет взаимно однозначное отображение на $\Gamma$ или ее часть, следовательно, с учетом (3)

$$
\begin{aligned}
\frac{1}{2 \pi} \int_{\Gamma} \frac{\partial g_{G}(\zeta, \infty)}{\partial n}|d \zeta| & \leqslant \sum_{j} \frac{1}{2 \pi} \int_{f\left(\gamma_{j}\right)} \frac{\partial g_{G}(\zeta, \infty)}{\partial n}|d \zeta|=\frac{1}{2 \pi} \sum_{j} \int_{\gamma_{j}} \frac{\partial g_{G}(f(\xi), \infty)}{\partial n}\left|f^{\prime}(\xi)\right||d \xi| \\
& \leqslant \frac{1}{2 \pi} \sum_{j} \sum_{k} \int_{\gamma_{j}} \frac{\partial g_{D}\left(\xi, z_{k}(\infty)\right)}{\partial n}|d \xi|=\sum_{k} \omega\left(z_{k}(\infty), \gamma, D\right) .
\end{aligned}
$$

Общий случай утверждения теоремы 2 следует теперь из инвариантности гармонической меры относительно дробно-линейных отображений.

ЗАмечание 1. Утверждение теоремы 2 справедливо и в случае, когда области $D$ и $G$ ограничены конечным числом жордановых дуг и имеют пустую внешность.

ЗАмечание 2 . В случае $D=\overline{\mathbb{C}} \backslash E, G=\overline{\mathbb{C}} \backslash F$, где $E$ и $F$ - системы нескольких отрезков действительной оси, утверждение теоремы 2 было анонсировано автором в [4].

ЗАмечАниЕ 3. Приведем пример, показывающий точность неравенства (3). Пусть $f(z)=z^{2}, D=\overline{\mathbb{C}} \backslash[-1,1], G=\overline{\mathbb{C}} \backslash[0,1], \gamma=\Gamma=[0,1]$. Тогда $\omega(\infty, \Gamma, G)=1, \omega(\infty, \gamma, D)=$ $1 / 2$.

Автор благодарен член-корреспонденту РАН В. Н. Дубинину за полезные замечания.

\section{СПИСОК ЦИТИРОВАННОЙ ЛИТЕРАТУРЫ}

[1] Р. Неванлинна, Однозначные аналитические функиии, ГИТТЛ, М.-Л., 1941. [2] С. Стоилов, Теория функиий комплексного переменного, т. 2, ИЛ, М., 1962. [3] В. Н. Дубинин, С. И. Калмыков, Матем. сб., 198:12 (2007), 37-46. [4] A. L. Lukashov, Inequalities for Derivatives of Rational Functions and Harmonic Measures, http:// www. sabanciuniv.edu/mdbf/analysis2007/documents/abstract_Lukashov_.pdf.

\section{А. Л. Лукашов}

Поступило

Саратовский государственный

17.02.2008

университет им. Н. Г. Чернышевского 